« Hervé Guay L'éveil culturel - Théâtre et presse à Montréal, 1898-1914 »

\author{
Jean-Marc Larrue \\ Theatre Research in Canada / Recherches théâtrales au Canada, Vol. 34, n 1, 2013, p. 139-142.
}

Pour citer ce chapitre :

LARRUE, Jean-Marc, « Hervé Guay L'éveil culturel - Théâtre et presse à Montréal, 1898-1914 », Theatre Research in Canada / Recherches théatrales au Canada, Vol. 34, n 1, 2013, p. 139-142. 
to make it live? And to allow oneself to live? Comment tuer Shakespeare, which reveals new gems on each rereading, offers fascinating insight into the works of Shakespeare, Chaurette's creative process, and the challenges of theatrical translation.

\section{HERVÉ GUAY}

L'éveil culturel - Théâtre et presse à Montréal, 1898-1914.

Montréal : Les Presses de l'Université de Montréal, 2010, 350 pp.

\section{JEAN-MARC LARRUE}

Issu d'une thèse de doctorat soutenue en 2005, l'essai Léveil culturel-Théâtre et presse à Montréal, 1898-1914 que Hervé Guay a publié aux Presses de l'Université de Montréal en 2010, se lit presque comme un roman, ce qui indique que cette mutation de la thèse vers l'essai, souvent hasardeuse, a ici parfaitement réussi. On apprécie la précision de l'information foisonnante et la langue élégante et fluide dans laquelle elle est livrée. Létude de Guay porte sur la critique théâtrale à une période qui est souvent considérée comme un premier âge d’or du théâtre à Montréal, qu'il soit anglophone ou francophone (ou même yiddishophone). C'est effectivement à la toute fin du XIX ${ }^{\mathrm{e}}$ siècle quapparaissent les premiers théâtres professionnels francophones dans ce qui était alors la métropole du Canada. En quelques années, les scènes se multiplient en se diversifiant et se spécialisant. Le cinéma, dont le succès ira croissant, n’a pas encore délogé le théâtre en tant que divertissement préféré des Montréalais, toutes langues confondues. Cette domination effervescente dure jusquau déclenchement de la Première Guerre mondiale qui ne met pas un terme à cette pratique multiple et ouverte, mais qui la déstabilise, la ralentit et force son repositionnement. Hervé Guay est donc tout à fait légitimé de se centrer sur cette période relativement brève mais d'une remarquable richesse.

Cette étude est à la fois nécessaire et exemplaire. Nécessaire parce quelle aborde un domaine généralement délaissé par les historiens du théâtre en dépit de son importance : la critique théâtrale. Précisons-le, ce désintérêt ne concerne pas que la période en question, elle est généralisée. Il était donc plus que temps que quelqu'un se penche sur cette composante essentielle de l'institution théâtrale et que Bourdieu intégrait à l'instance de légitimation, capitale dans la logique de la distinction et tente, par une analyse minutieuse et approfondie, d'en révéler les mécanismes, 
les enjeux, les valeurs et les agents. Létude de Guay est d'autant plus méritoire que la pratique critique, en cette période pionnière, se caractérise d’abord par son instabilité : les périodiques et les journaux, où se concentre l'essentiel de l'activité, ont généralement une durée de vie assez courte. Quant aux " critiques », dont l'identité est souvent masquée par un pseudonyme quand ce n'est pas derrière l'anonymat, ils ont, en général, des carrières désespérément éphémères.

Lexemplarité de louvrage provient de la rigueur, la transparence et l'intégrité de la démarche. Il comporte essentiellement deux parties. La première porte sur les productions critiques (écrites et visuelles), la seconde sur la carrière et la production de sept critiques particuliers. D’entrée de jeu, Hervé Guay présente ses choix et ce qui les motive. Il se limite volontairement à des hebdomadaires parce qu'il considère que c'est là, plutôt que dans les grands quotidiens de l'époque, que s'exprime le mieux la pratique critique. Il retient quatorze " publications ayant paru au moins douze mois consécutifs " (14) : L'Action, L'Autorité, Le Bulletin, Le Canadien, La Croix, Les Débats, Le Monde illustré, Le Nationaliste, Le Passe-Temps, Le Pays, La Petite Revue, le Rappel, Le Réveil et Le Samedi. Quant aux sept critiques dont it traite dans la seconde partie, ils ont en commun d'avoir « couvert au minimum léquivalent d'une saison théâtrale au cours de cette période » (14). Il s’agit de Gustave Comte, Frédéric Pelletier, Jules Jéhin-Prume, Marcel Dugas, Ernest Tremblay, Roger Valois et Joseph Baril.

On peut être en accord ou en désaccord avec ces divers choix, mais ils ont l'avantage dêtre limpides. Ayant beaucoup travaillé dans la presse de cette époque, je métonne un peu de l'affirmation justificative selon laquelle « [c]e sont les hebdomadaires généralistes et non les quotidiens [dont La Presse et La Patrie, pour ne parler que des quotidiens français] qui constituent la frange des journaux où l'on s'intéresse le plus activement à l'activité théâtrale » (12). Ce n'est pas l'impression que j'en avais, ni d'un point de vue qualitatif, ni d'un point de vue quantitatif, ni de celui de la régularité de parution des informations. Mais Guay a un argument de poids. Son « étude peut sembler accorder une importance démesurée à des journaux peu influents " (13), mais, rappelons-le, son projet est de brosser le tableau le plus complet possible de la pratique critique de lépoque, non d'en mesurer l'influence. Et la critique des grands quotidiens, malgré la richesse des données quelle diffuse, ne couvre qu'un champ limité de cette pratique. Guay a sûrement raison d'affirmer que, soucieux d'at- 
tirer le plus large lectorat possible, « les quotidiens relèguent les sujets les plus conflictuels au second plan au profit de questions plus consensuelles » (37).

À vrai dire, cela serait effectivement démesuré et disqualifierait l'ensemble de la démarche si la pratique critique telle qu'on peut l'observer dans ces publications, souvent artisanales et au rayonnement limité, était radicalement différente de ce que présente la presse à grand tirage des quotidiens de l'époque et des époques ultérieures. En fait, le type de textes que publie la presse quotidienne se trouve également dans les hebdomadaires. La grande vertu de travail est donc de fournir des données factuelles, des outils conceptuels et des outils méthodologiques applicables à tout travail critique, quelle que soit l'époque ou la publication concernée. On regrette cependant que le fait de s’en tenir aux périodiques ne permette pas à Guay de suivre les parcours des critiques qui passent des hebdomadaires aux quotidiens. Ces déplacements sont eux-mêmes très significatifs en ce sens qu'ils permettent de mesurer l'influence relative de chacun des critiques. Une plus grande ouverture à la presse quotidienne, ou au moins un assouplissement de certains critères, aurait aussi eu pour effet d'inclure des femmes à la sélection des auteurs retenus, ce qui aurait enrichi la recherche.

Dans la première partie, Guay brosse un inventaire détaillé des différents types d'intervention qu'effectuent les critiques dans les quatorze hebdomadaires retenus. Il regroupe ces interventions ou " genres " (la critique de représentation, la caricature (visuelle), la photo, le dessin d'artiste, le pêle-mêle, la brève, le bilan, etc.) selon cinq grandes catégories : les genres informatifs, les genres publicitaires, les genres du divertissement, les genres argumentatifs, les genres composites. Mais Guay ne s'en tient pas à un simple exercice taxonomique, il explique le rapport de chacun de ces genres à la dynamique de lépoque marquée par la concurrence des périodiques et des théâtres, mais aussi par des questions idéologiques. On comprend ainsi pourquoi certains périodiques privilégient tel genre plutôt que tel autre. Lanalyse est convaincante et, encore une fois, Guay fournit des informations précieuses, aussi bien au lecteur ordinaire quaux historiens.

La seconde partie est d'un tout autre ordre. En fonction des critères qu'il sétait fixés, Guay retient sept critiques qui ont donc couvert l'équivalent d'au moins une saison au cours de la période dans les périodiques sélectionnés. Létude bifurque ici sur les questions d'identité culturelle. Lapproche est fine. Puisqu'il apparait que les sept critiques désirent tous que davantage de specta- 
teurs (francophones) aillent au théâtre parce que, en plus de divertir, le théâtre contribue à l'affirmation de l'identité collective (canadienne-française), l’examen de leurs textes devrait révéler les caractéristiques dominantes de cette identité. Guay applique donc, aux textes de ces critiques, une grille unique d'analyse en fonction de quatre axes : la catholicité, la modernité (nous sommes en plein avènement de la modernité théâtrale), la francité et l'américanité. Là encore, on pourrait chicaner. Pourquoi la moralité ou l'esthétique ou l'art ne seraient-ils pas des catégories à part ? Ceci dit, le résultat est étonnant. La grille révèle une variété de tendances inattendue. Au-delà des convictions idéologiques et des préférences esthétiques qui en découlent, il existe de véritables pensées critiques, solidement articulées et très judicieusement exprimées. Certains défendent la primauté de l'influence française (francité) et considèrent l'américanité dangereuse (à cause des dangers d'assimilation), d'autres voient au contraire l'américanité comme une valeur essentielle de l'identité des francophones d'Amérique. La modernité a, de même, ses détracteurs et ses apôtres, et la catholicité cristallise tout ce qui relève de la moralité et de l'influence-complexe-du clergé catholique. Le tableau est passionnant et, là encore, déborde du cadre théâtral pour révéler une société en pleine turbulence. Comme dans la première partie, Guay ne se contente pas de structurer les discours en fonction des quatre axes, mais il montre comment ils interagissent les uns avec les autres.

Louvrage de Hervé Guay est une contribution importante à notre connaissance de l'histoire du théâtre au Québec. Il n'en révèle pas seulement des aspects marquants et méconnus, il fournit des outils durables pour mieux les explorer. 